\title{
Transforming Diabetes Care Through Artificial Intelligence: The Future Is Here
}

\author{
Irene Dankwa-Mullan, MD, MPH, Marc Rivo, MD, MPH, Marisol Sepulveda, DO, MPH, \\ Yoonyoung Park, $\mathrm{ScD}^{4}$, Jane Snowdon, $\mathrm{PhD}^{5}$, and Kyu Rhee, MD, MPP ${ }^{6}$
}

\begin{abstract}
An estimated 425 million people globally have diabetes, accounting for $12 \%$ of the world's health expenditures, and yet 1 in 2 persons remain undiagnosed and untreated. Applications of artificial intelligence (AI) and cognitive computing offer promise in diabetes care. The purpose of this article is to better understand what AI advances may be relevant today to persons with diabetes (PWDs), their clinicians, family, and caregivers. The authors conducted a predefined, online PubMed search of publicly available sources of information from 2009 onward using the search terms "diabetes" and "artificial intelligence." The study included clinically-relevant, high-impact articles, and excluded articles whose purpose was technical in nature. A total of 450 published diabetes and AI articles met the inclusion criteria. The studies represent a diverse and complex set of innovative approaches that aim to transform diabetes care in 4 main areas: automated retinal screening, clinical decision support, predictive population risk stratification, and patient self-management tools. Many of these new AIpowered retinal imaging systems, predictive modeling programs, glucose sensors, insulin pumps, smartphone applications, and other decision-support aids are on the market today with more on the way. AI applications have the potential to transform diabetes care and help millions of PWDs to achieve better blood glucose control, reduce hypoglycemic episodes, and reduce diabetes comorbidities and complications. AI applications offer greater accuracy, efficiency, ease of use, and satisfaction for PWDs, their clinicians, family, and caregivers.
\end{abstract}

Keywords: diabetes care, artificial intelligence, cognitive computing, artificial pancreas, retinal imaging, glucose monitoring

\section{Background}

D IABETES is a Global PANDEMic. An estimated 425 million people worldwide have diabetes, accounting for $12 \%$ of the world's health expenditures, and yet 1 in 2 persons remain undiagnosed and untreated. ${ }^{1}$ Type 2 diabetes is driven by the global obesity epidemic and a sedentary lifestyle that overwhelms the body's internal glucose control requiring exogenous insulin. ${ }^{2}$ Millions of newborns are born to mothers with gestational diabetes. Children born with type 1 diabetes mellitus, in which the body cannot produce insulin, require life-long insulin therapy. In the United States, diabetes is the leading cause of kidney failure, lower limb amputations, adult-onset blindness, and almost doubles the risk of heart attack and all-cause mortality, leading to hospitalization, long-term complications, and higher costs. ${ }^{3}$

Decades of well-designed studies have established that intensive therapy effectively delays the onset and slows the progression of diabetes-related complications, such as retinopathy, nephropathy, and neuropathy. ${ }^{4}$ Yet, a recent study of 300,000 patients with type 2 diabetes who were started on medical therapy found that after 3 months, $31 \%$ of patients had discontinued their diabetes medications altogether: this increased to $44 \%$ by 6 months, and to $58 \%$ by 1 year. Only $40 \%$ eventually restarted diabetes medications. ${ }^{5}$ Optimal care for persons with diabetes (PWDs) often is hampered by

\footnotetext{
${ }^{1}$ IBM Corporation, Watson Health, Bethesda, Maryland.

${ }^{2}$ Population Health Innovations, Inc., Miami Beach, Florida.

${ }^{3}$ Occupational Medicine, University of Iowa, Iowa City, Iowa.

${ }_{5}^{4}$ IBM Corporation, IBM Research, Cambridge, Massachusetts.

${ }_{5}^{5}$ IBM Corporation, Watson Health, Yorktown Heights, New York.

${ }^{6}$ IBM Corporation, Watson Health, Cambridge, Massachusetts.
}

(C) Irene Dankwa-Mullan et al. 2018; Published by Mary Ann Liebert, Inc. This Open Access article is distributed under the terms of the Creative Commons License (http://creativecommons.org/licenses/by/4.0), which permits unrestricted use, distribution, and reproduction in any medium, provided the original work is properly cited. 
the absence of real-time, key health information necessary to make informed choices associated with intensive therapy and tight diabetes control. Although advances in technology offer unprecedented and inexpensive access to essential information for many individuals in many fields, its impact in the care of patients with diabetes seems rather limited. The challenges of real-time diabetes care information are compounded by the rapid expansion of medical knowledge. The index of biomedical literature contains more than 28 million articles as of June 2018 and is growing at a rate of more than 850,000 new citations each year. ${ }^{6}$ Each person will generate more than 1 million gigabytes of health-related data in his or her lifetime, the equivalent of about 300 million books. An estimated $80 \%$ of health data is unstructured. This includes clinician notes, clinical trials, hospital records and discharge summaries, imaging and laboratory reports, and nonclinical data sources, including device and sensor data (often referred to as Internet of Things data), genomic data, and social determinants of health data. ${ }^{7}$ Ninety percent of a person's health outcomes may be attributed to genomics and exogenous data, underscoring the importance of PWDs and their clinicians collecting and leveraging these data to make informed health choices. ${ }^{8}$

Rapid advances in artificial intelligence (AI) offer the promise of making both real-time structured and unstructured health data available for the care of PWDs. The Turing Archive for the History of Computing defines AI as "the science of making computers do things that require intelligence when done by humans." 9 AI covers a broad range of approaches to simulating human intelligence and performing various reasoning tasks, such as visual perception, speech recognition, analytics, decision making, and translation between languages. Cognitive systems employ the spectrum of AI approaches to extend and scale human knowledge and expertise by enabling humans to leverage vast knowledge sources rapidly to solve problems.

Today, AI is harnessing massive amounts of vital information to meet consumer demand in every business, including health care. A 2017 survey found that $68 \%$ of mobile health app developers and publishers believe that diabetes continues to be the single most important health care field with the best market potential for digital health solutions within the near future, and that $61 \%$ see AI as the most disruptive technology shaping the digital health sector. ${ }^{10}$ Although advances in AI for health care are being reported in the literature ${ }^{11}$ and new AI-powered devices are being approved for diabetes care, ${ }^{12}$ a systematic review of clinically relevant diabetes AI applications is missing. The purpose of this article is to better understand what meaningful AI advances may be relevant today to PWDs, their primary care clinicians, endocrinologists, health professionals, family, and caregivers.

\section{Methods}

The study team conducted a predefined, online PubMed search of publicly available sources of information using the search terms "diabetes" and "artificial intelligence (AI)." To identify articles with clinically-relevant, high-impact diabetes AI applications, the team excluded manuscripts with publication dates before 2009 and those whose purpose was primarily technical in nature (eg, focused solely on AI algorithm development). The first-pass search identified a total of 763 clinically-relevant abstracts. Additional review excluded 313 as duplicative or primarily technical. The second-pass review yielded a total of 450 unique, clinicallyrelevant articles researching the direct application of AI in diabetes prevention, diagnosis, and treatment. The information was then collated and classified. The research was conducted between March and May of 2018.

\section{Results}

The PubMed search yielded a total of 450 clinically-relevant and high-impact articles published in the last decade related to the field of applied AI in diabetes care. The AI applications aimed to improve a broad spectrum of diabetes care, from diabetes screening and detection to monitoring and treatment, and included apps, devices, and systems that aid patients, clinicians, and health systems. The published articles included in this search were of high clinical impact in that they sought to produce and test AI approaches that may impact diabetes care significantly in the areas of access, accuracy, efficiency, affordability, speed, and satisfaction of patients, clinicians, and caregivers. A review of the high-impact articles suggests that AI applications are aiming to transform diabetes care in 4 main areas: automated retinal screening, clinical decision support, predictive population risk stratification, and patient self-management tools, as summarized in Table 1.

A diverse and complex set of AI approaches and cognitive computing systems were employed in these studies. Table 2 defines the more common AI approaches described in the research and lists their clinical applications in diabetes care.

Table 1. Categorization of Artificial Intelligence and Diabetes Care

\begin{tabular}{|c|c|c|}
\hline Category & Number of articles & Most common clinical AI applications \\
\hline Automated Retinal Screening & 96 & $\begin{array}{l}\text { Detection of diabetic retinopathy, maculopathy, exudates, } \\
\text { and other abnormalities from normal findings }\end{array}$ \\
\hline Clinical Decision Support & 126 & $\begin{array}{l}\text { Detection and monitoring of diabetes and comorbidities } \\
\text { such as neuropathy, nephropathy and wounds }\end{array}$ \\
\hline Predictive Population Risk Stratification & 135 & $\begin{array}{l}\text { Identification of diabetes subpopulations at higher risk for } \\
\text { complications, hospitalization, and readmissions }\end{array}$ \\
\hline Patient Self-Management Tools & 94 & $\begin{array}{l}\text { AI-improved glucose sensors, artificial pancreas, activity } \\
\text { and dietary tracking devices }\end{array}$ \\
\hline TOTAL & 450 & \\
\hline
\end{tabular}

AI, artificial intelligence. 


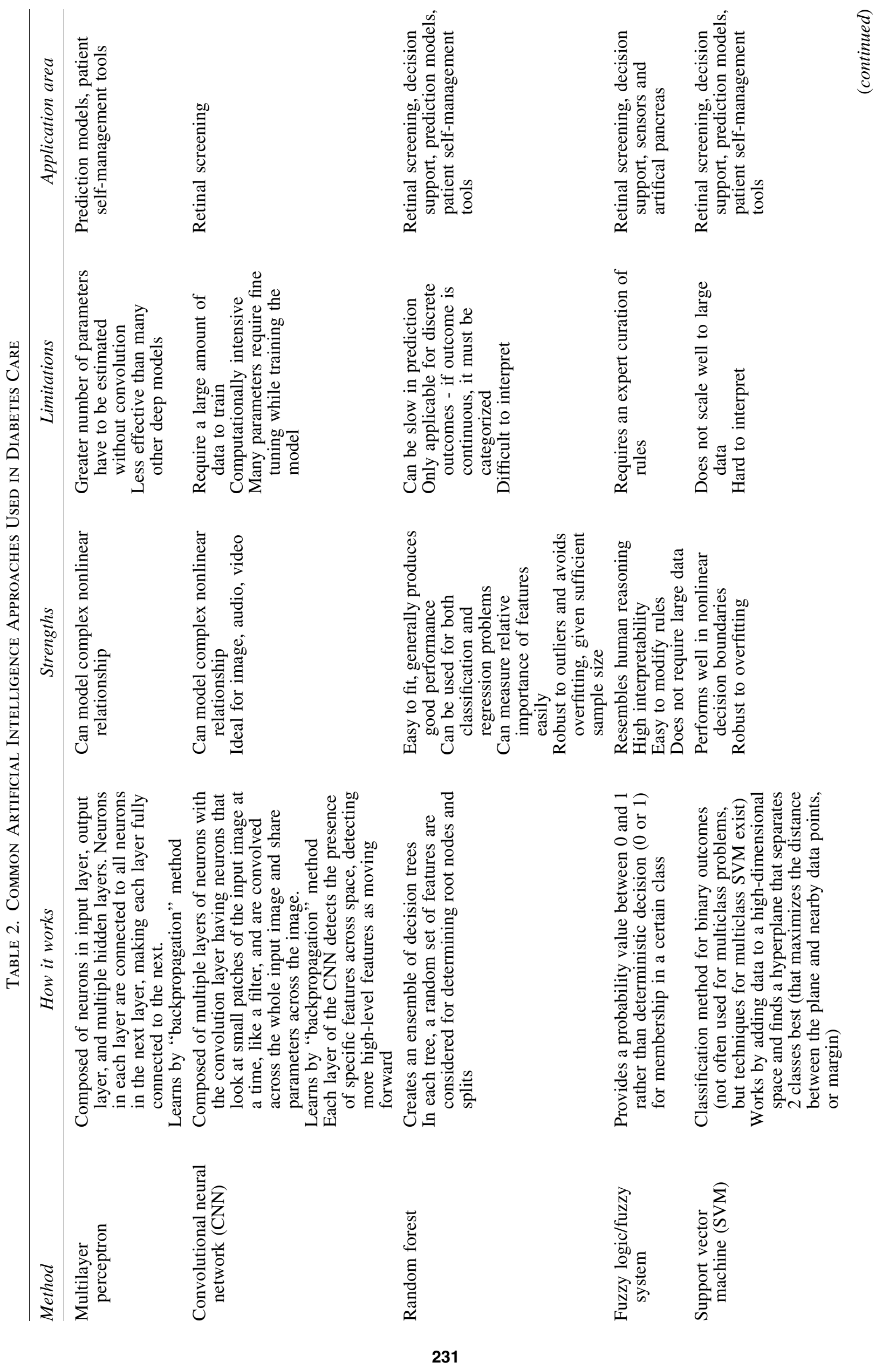




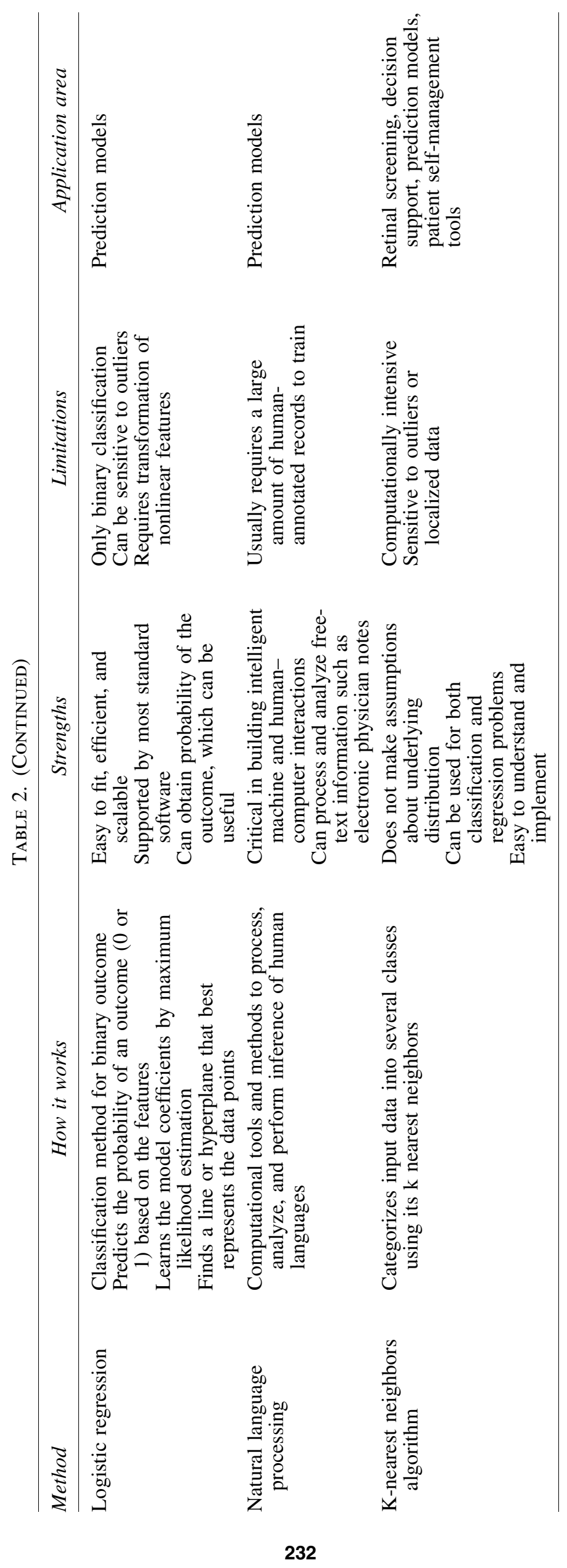




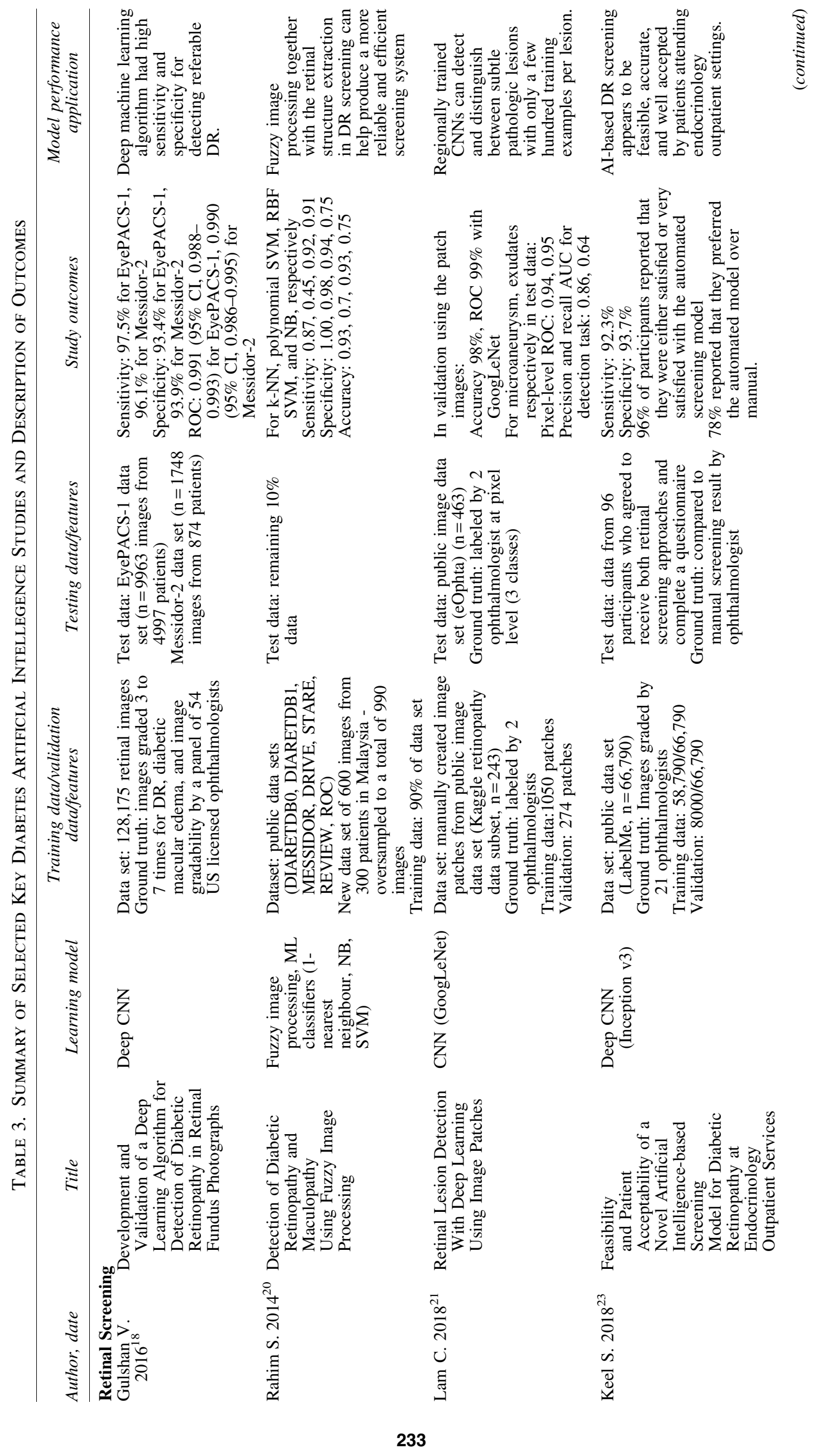



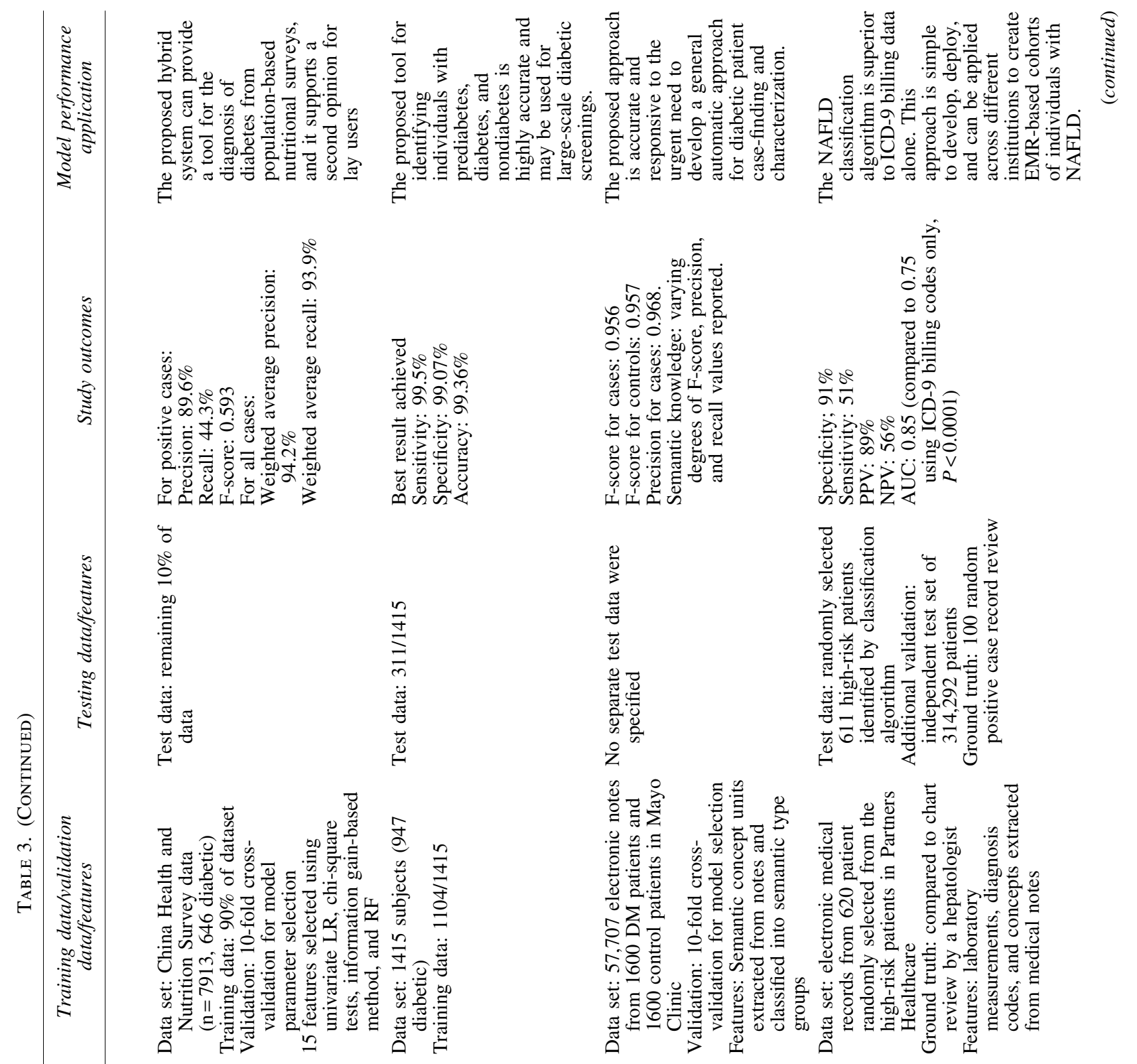

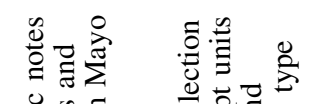
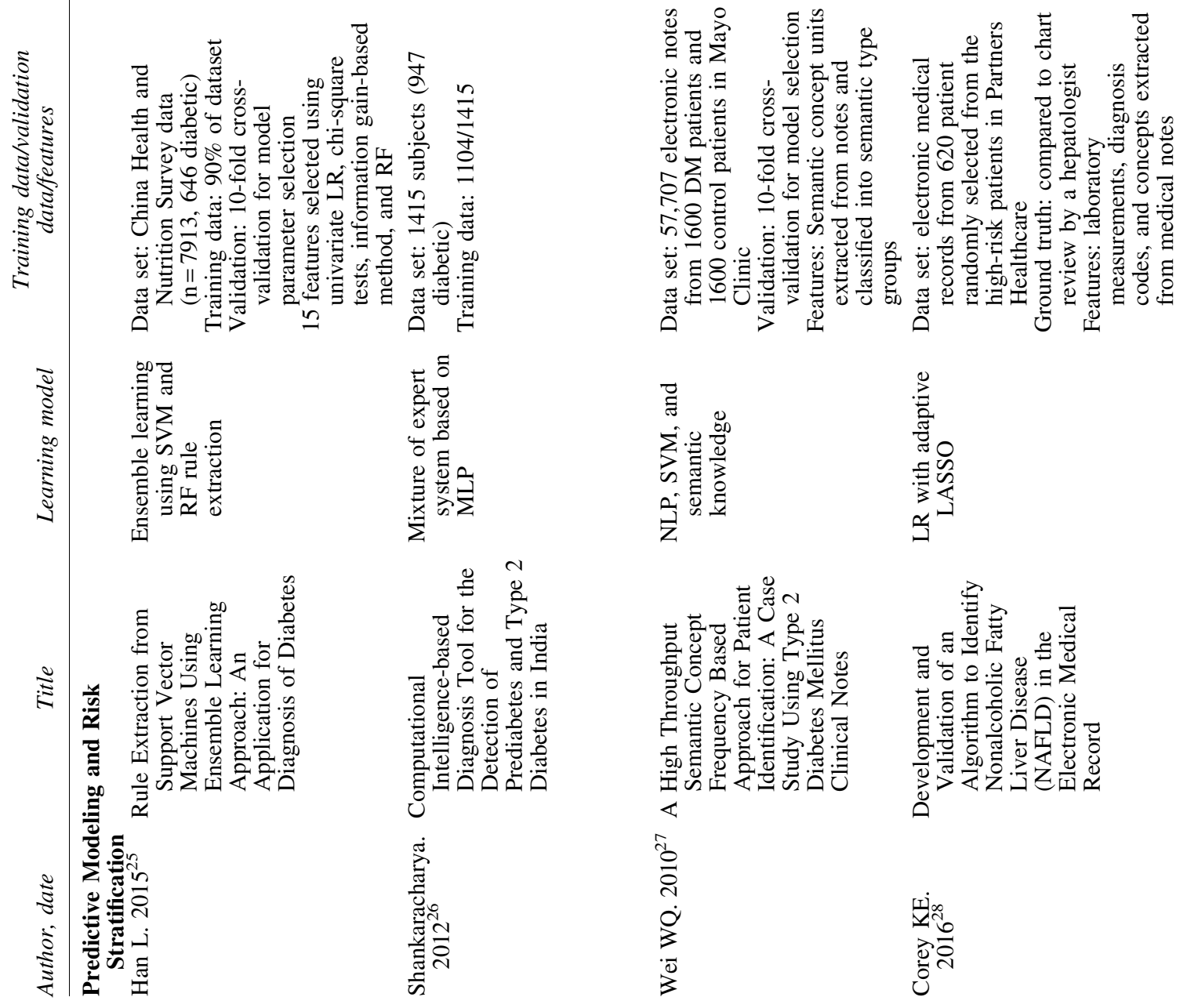

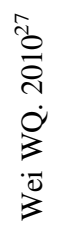

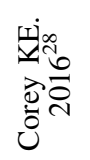




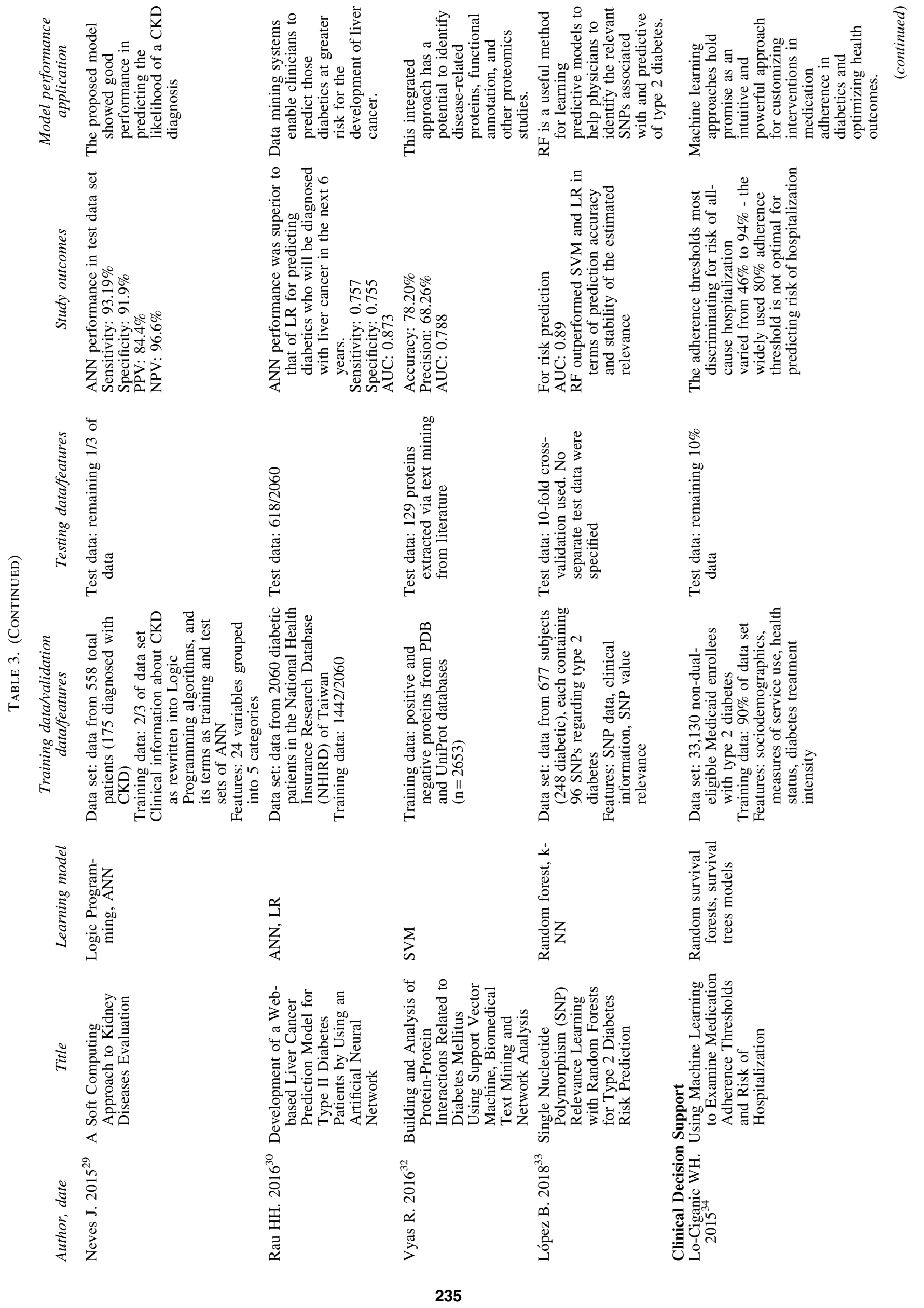




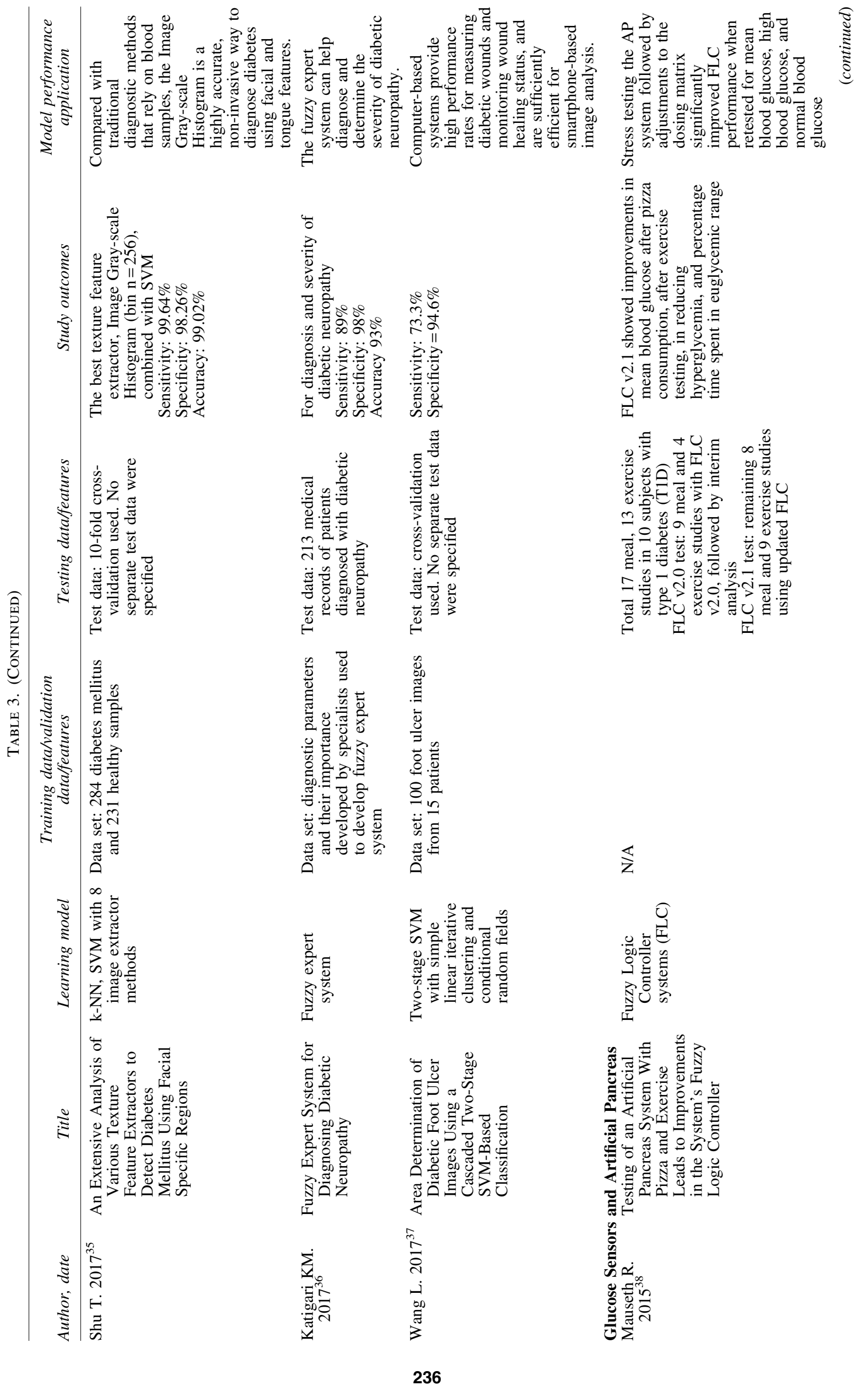




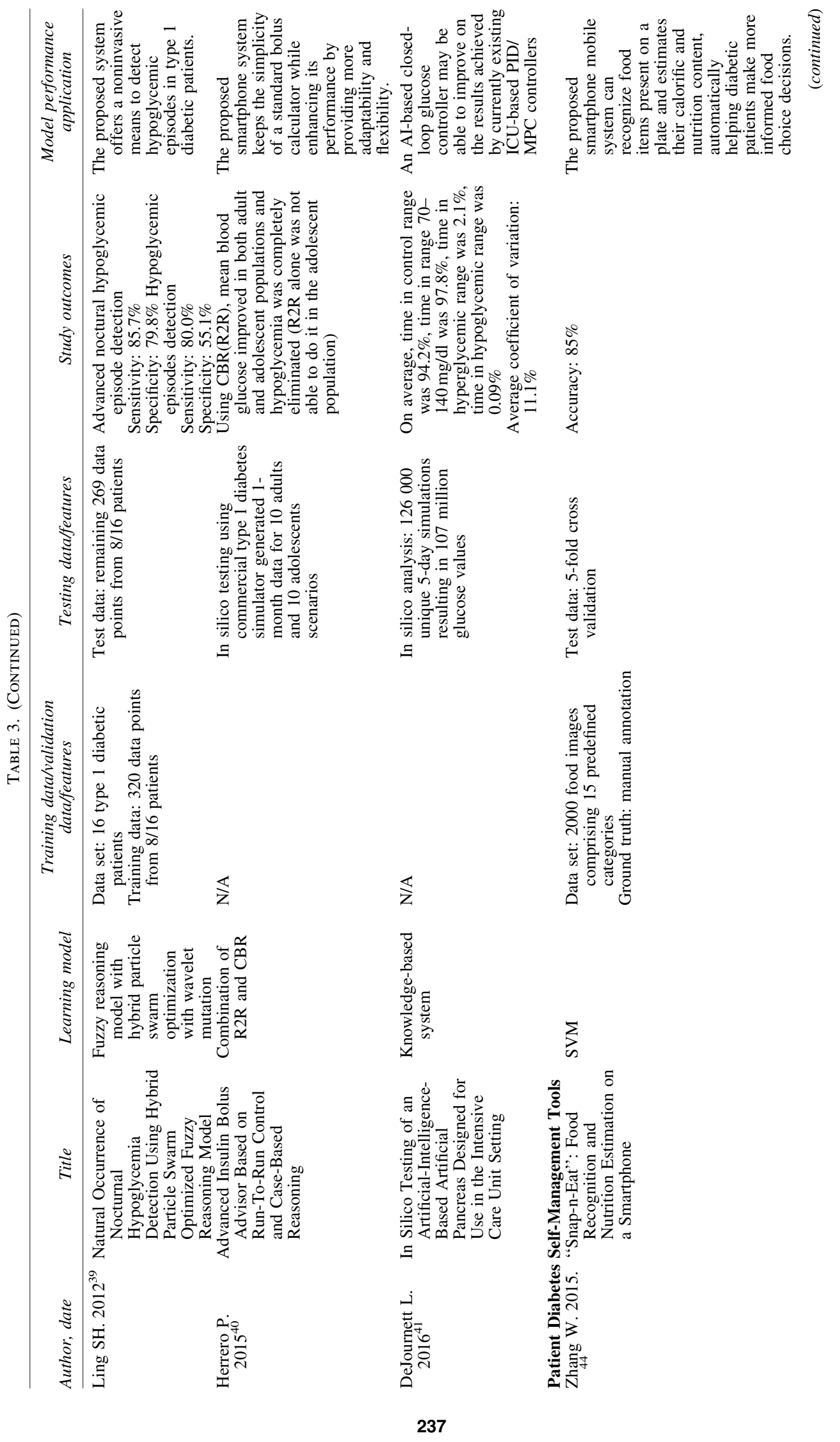




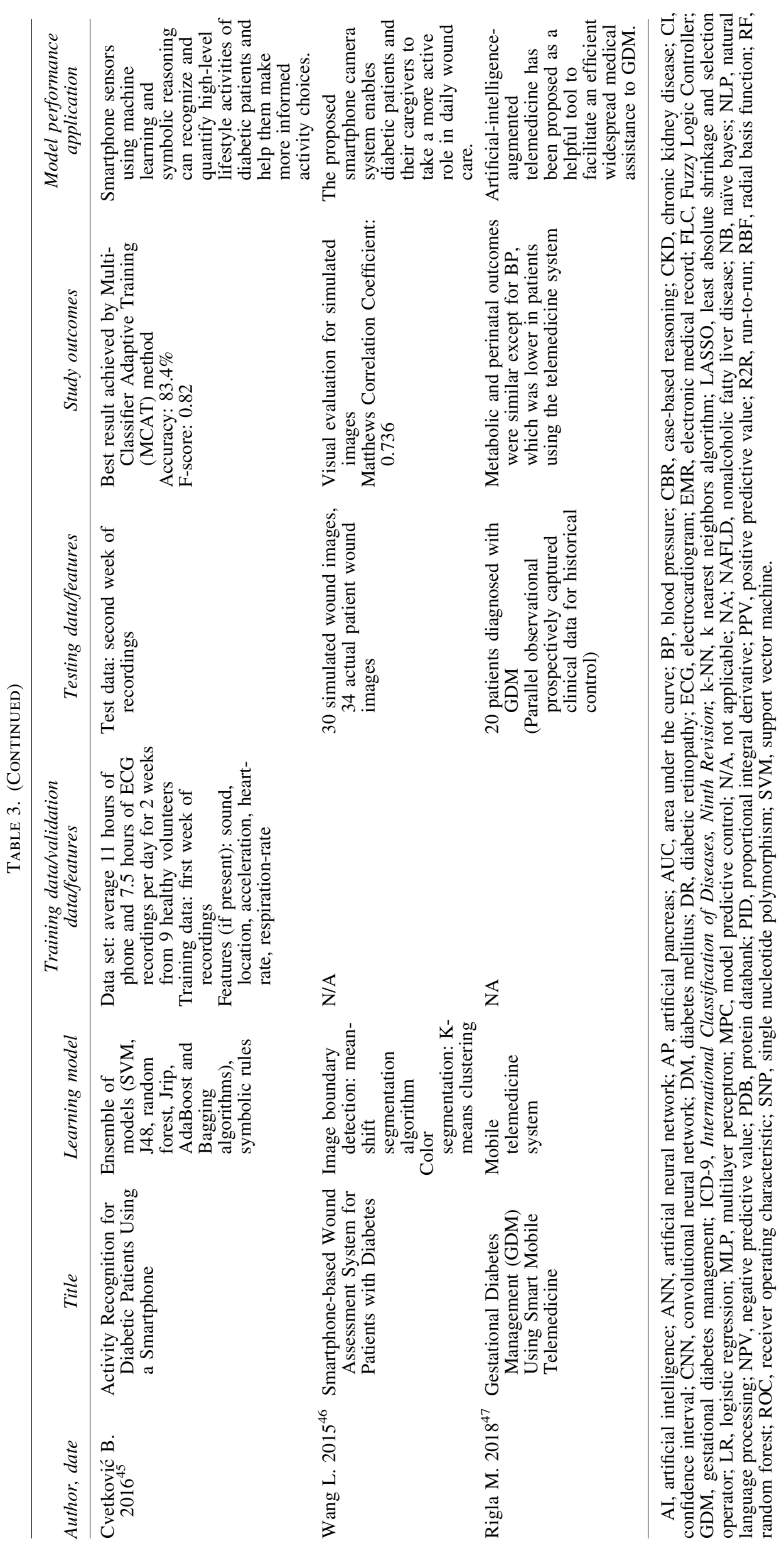


A review of the published articles documented the substantial advances in AI technology over the last 10 years and how it is helping PWDs and their clinicians make more informed choices. Examples of the most common AIpowered diabetes care devices and systems identified in the published literature are summarized in Table 3 . Table 3 provides examples of the research questions receiving the most attention among diabetes AI researchers and product developers over the past decade.

\section{Discussion}

The published studies suggest that a broad spectrum of market-ready AI approaches are being developed, tested, and deployed today in the prevention, detection, and treatment of diabetes. The total number of published technical articles reporting advances in the field of diabetes and AI increased exponentially in the past decade, from 2600 in 2008, to 5500 in 2013, to more than 10,000 in $2017 .{ }^{13}$ Millions of patient health records and newly published research exist that need to be further processed, analyzed, and learned from to create a current diabetes knowledge base for patients, researchers, doctors, and clinicians. Because of AI's ability to rapidly interpret and process enormous amounts of data into simple actionable guidance, these published studies suggest that AI has significant potential to improve screening, diagnosis, and management of patients with diabetes. ${ }^{14}$

Researchers are employing various AI approaches to interpret the vast amount of relevant data that need to be analyzed and assessed. ${ }^{15}$ Table 2 describes the more common AI approaches described in the research and lists their clinical applications in diabetes care. AI involves a wide spectrum of increasingly complex algorithms encompassed within the terminology of machine learning, deep learning, and cognitive computing. In machine learning, experts typically "train" AI systems with large amounts of data and algorithms, which enable the machine to examine relationships and learn from them. In deep learning, AI systems identify relevant insights for diagnostic support, while automatically conducting certain complex and time-consuming tasks. Cognitive AI systems go even further by understanding, reasoning, interacting, and learning. These systems understand by processing and deeply interpreting the available data, both structured and unstructured, at enormous speed and volume. They reason by understanding entities and relationships, making connections, proposing hypotheses, and evaluating evidence. In contrast to the electronic health record, they provide a more natural interaction between human and computer, facilitating dialogue, visualization, and collaboration. ${ }^{16}$ Cognitive AI Systems learn by collecting and evaluating feedback at all levels of the system. The result is practical knowledge, aids, and devices for diabetes patients and their clinicians that save time, improve efficiency, enhance clinical decision making, empower patients, and have the potential to improve health outcomes and patient and clinician satisfaction. Although a more complete explanation of AI is beyond the focus of this research article, this study indicates that $\mathrm{AI}$ is a growing presence in diabetes care with the potential to transform millions of people's lives.

Today, research suggests that AI approaches are rapidly transforming care in 4 vital areas: improved screening and detection of diabetic retinopathy (DR) and macular edema; individualized predictive risk stratification and treatment; decision-support tools for clinicians; and patient selfmanagement aids. Key examples from the published literature are summarized in Table 3 and follow.

DR is the most serious cause of secondary blindness, exacting an enormous burden on individuals, families, and the health care system. The annual diabetic retinal exam serves to screen and proactively detect diabetes patients with early treatable retinopathy. It is estimated that $98 \%$ of vision loss from DR and macular edema is avoidable through improved prediction, early detection, and treatment strategies, and its cost-effectiveness is well established. ${ }^{17}$ Yet, major barriers to implementing more widespread screenings include the limited number of eye care practitioners who are trained in interpreting retinal images, along with access to care barriers.

Today, research documents that deep learning-based AIgrading of DR from retinal photographs is associated with sensitivity and specificity over $90 \% .^{18-21}$ Recently, the US Food and Drug Administration (FDA) approved marketing the first medical device to use AI to screen diabetes patients for retinopathy. ${ }^{6}$ The device, called IDx-DR (IDx LLC, Coralville, IA), is a software program that uses an AI algorithm to analyze images of the eye taken with a retinal camera called the Topcon NW400 (Topcon Medical Systems, Inc., Oakland, NJ). Digital images of the patient's retinas are uploaded to a cloud server on which IDx-DR software is installed. If the images are of sufficient quality, the software provides the doctor with one of 2 results: (1) "more than mild diabetic retinopathy detected: refer to an eye care professional" or (2) "negative for more than mild diabetic retinopathy; rescreen in 12 months." IDx-DR is the first device authorized for marketing that provides a screening decision without the need for a clinician to also interpret the image or results. ${ }^{22}$ These automatic systems enable non-eye health professionals in primary care physician offices to perform on-site retinal screening and provide on-the-spot normal results or immediate referrals to the eye specialist without the need for eye specialists, with significantly higher patient satisfaction with the simplified process. ${ }^{23,24}$

Today, AI-driven predictive modeling proactively identifies diabetes populations with the highest risks of avoidable complications resulting in unnecessary emergency department visits, admissions, and readmissions. ${ }^{25}$ Larger physician groups, health care systems, and health plans utilize AI to "mine" large sets of digital and unstructured patient data to proactively identify and characterize diabetes populations, ${ }^{26,27}$ find patients at risk for diabetic comorbidities, ${ }^{28-30}$ identify patients for special diabetes disease management programs, ${ }^{31}$ and discover relevant proteins $^{32}$ and genes ${ }^{33}$ associated with and predictive of diabetes.

Today, AI provides practice decision-support tools for physicians and other health professionals caring for PWDs. Machine learning approaches help physicians customize diabetes medications to optimize adherence and health outcomes. ${ }^{34}$ AI-powered devices help physicians diagnose diabetes noninvasively, ${ }^{35}$ and more accurately measure and monitor the severity of diabetic neuropathy ${ }^{36}$ and diabetic wounds. ${ }^{37}$

Today, research suggests that diabetes management for both PWDs and their clinicians is being simplified and improved by new sensors, pumps, smartphone applications, and other breakthroughs in AI to achieve better blood glucose control, ${ }^{38}$ reduce hypoglycemic episodes, ${ }^{39}$ and 
improve patient satisfaction and reported outcomes. ${ }^{40} \mathrm{~A}$ 2017 meta-review of published clinical trials of the latest, automated, personal or real-time continuous glucose monitoring devices (RT-GCM) using computerized AI algorithms concluded that a wide range of AI-powered RT-CMG devices are entering the market to enable PWDs and their clinicians to assess and improve glycemic control, reduce hypoglycemic episodes, especially at night, and to improve A1c levels.

Published research documents the extensive testing under way with the "artificial pancreas," known also as a Closed Loop System, which combines continuous glucose measurement with algorithm-driven insulin pumps to reduce hypoglycemia and improve diabetes self-care. ${ }^{41}$ The latestgeneration sensors, which are more accurate and sensitive for hypoglycemia, and the development of algorithms that allow insulin infusion to be suspended during hypoglycemia and glucagon to be administered, provide a safe and effective system for persons at high risk of hypoglycemia. A meta-review of 12 published transition and home studies of 10 to 58 patients comparing clinical performance and patient acceptance of Artificial Pancreas Devices (APDs) with traditional monitoring concluded that research, testing, and validation has moved from the laboratory to free-living, unsupervised home settings in the past decade, with accuracy and reliability of the latest APD devices compatible with safe operation and high patient satisfaction. ${ }^{42} \mathrm{~A}$ recent review identified 18 closed-loop APDs being tested - 6 APDs in the home setting, 5 in outpatient settings, and 7 in inpatient settings - with planned commercial availability in 2018 and $2019 .^{43}$

Today, research findings document the promise of diabetes apps to assist users in tracking and analyzing their data in a hassle-free way and to deliver personalized data-driven insights that PWDs may apply in their daily life. Today, best-in-class apps provide comprehensive nutrition databases that tell a user the nutritional content after scanning the barcode, allow them to search for restaurant menu items or popular meals by their names, or recognize food items on a plate. ${ }^{44}$ Smartphone sensors using machine learning and symbolic reasoning can recognize and quantify high-level lifestyle activities of patients with diabetes and help them make more informed activity choices. ${ }^{45}$ An AI smartphone camera system enables patients with diabetes and their caregivers to take a more active role in daily wound care, and may potentially accelerate wound healing, save travel cost, and reduce health care expenses. ${ }^{46}$ AI-augmented telemedicine has been studied to facilitate medical assistance in the homes of pregnant women with gestational diabetes, with a high degree of patient acceptance. A research study incorporated computer-interpretable clinical practice guidelines, and access to data from the electronic health record as well as from glucose, blood pressure, and activity sensors. $^{47}$

On March 14, 2018, the FDA approved Medtronic's Guardian Connect, the first AI-powered continuous glucose monitoring (CGM) system, for use in PWDs between the ages of 14 and 75 years. Guardian Connect utilizes a predictive algorithm that alerts patients of significant swings in blood glucose levels up to 60 minutes prior to the event. When combined with the Guardian Sensor 3, which is placed on the abdomen to monitor blood glucose levels every 5 minutes and sent to a personal app, the Guardian Connect system was accurate and was able to alert patients of about $98.5 \%$ of hypoglycemic events so that they could proactively take action to normalize blood sugar. ${ }^{48}$

This information also can be shared and monitored with caretakers and family members in real time or via text message. In addition, Guardian Connect CGM is connected to the Sugar.IQ smart diabetes assistant. Utilizing AI technology from IBM Watson Health, the Sugar.IQ assistant continually analyzes how a patient's blood glucose levels respond to factors such as food intake, insulin dosages, and daily routines. Relative to baseline metrics, Sugar.IQ conferred in 256 Guardian Connect users tested a 36-minute/ day improvement in blood glucose time-in-range or 9 full days a year, a 30-minute/day decrease in time $>180 \mathrm{mg} / \mathrm{dl}$, and a 6-minute/day decrease in time $<70 \mathrm{mg} / \mathrm{dl}$, all statistically significant. During the course of the $31+$ patient-years of use, Sugar.IQ generated 655 insights for PWDs related to hypoglycemia and 699 related to hyperglycemia. In addition, 134 Sugar.IQ users were randomly given Fitbits exercise monitoring applications during the course of the study. Results showed that glucose responses to meals and activity vary greatly, demonstrating the importance of personalization in diabetes self-management. Notably, 231 of the $256(90 \%)$ users recorded at least 2 weeks of data, demonstrating a solid pattern of engagement with the AIpowered diabetes self-management application. ${ }^{49}$

Many challenges remain before diabetes AI apps, devices, and systems become ubiquitous in the health care marketplace. One major challenge is technical interoperability between systems: the ability of 2 or more systems to exchange and use the information. ${ }^{50}$ In addition, expensive upfront and ongoing costs, physician cooperation, and the complexity of meeting Meaningful Use criteria stifle adoption and innovation. ${ }^{51}$

Another major challenge is the limitation in reproducing AI results from published studies. A most basic problem is that researchers often do not share their source codes, sometimes for competitive reasons. A survey of 400 algorithms presented in papers at 2 top AI conferences in the past few years revealed that only $6 \%$ of the presenters shared the algorithm's code. Only one third shared the data used to test their algorithms, and just half shared the "pseudocode"-a limited summary of a source code algorithm. ${ }^{52}$ In addition, assuming one can obtain and run the original pseudocode, it still might not do what is expected. In the area of AI called machine learning, in which computers derive expertise from experience, the training data for an algorithm - for example, the key information to train speech-recognition learning systems - also can influence its performance.

Despite these challenges, this review of recentlypublished, high-impact, and clinically-relevant studies suggests that diabetes is attracting top health care technology companies as well as start-ups that are using innovative AI technologies and approaches to tackle daily challenges faced by PWDs. Many of the applications have received regulatory approval in the past few years and are on the market today. Many more are on the way with the aim to disrupt and transform diabetes care by improving accuracy, efficiency, ease of use, simplicity, and enjoyment on behalf of PWDs and their providers, caregivers, and family. The 
published literature suggests that the combination of continuous monitoring and real-time feedback to PWDs may be able to identify meaningful patterns and lead to personalized insights that increase patient and clinician engagement, confidence, and success in maintaining blood glucose levels under better control.

\section{Acknowledgment}

The authors would like to acknowledge the important contributions of Lisa Latts, MD, MSPH, MBA, to this manuscript and the field of artificial intelligence and health care transformation.

\section{Author Disclosure Statement}

Drs. Dankwa-Mullan, Park, Snowdon, and Rhee declare that there are no conflicts of interest. Drs. Rivo and Sepulveda received consulting fees from IBM Watson Health during the conduct of the study. This research did not receive any specific grant from funding agencies in the public, commercial, or not-for-profit sectors. The views expressed in this article are the authors' own and not an official position of IBM.

\section{References}

1. International Diabetes Federation (IDF). IDF diabetes atlas, 7th edition. Brussels, Belgium: International Diabetes Federation, 2015.

2. Liu J, Wang R, Ganz ML, Paprocki Y, Schneider D, Weatherall $\mathrm{J}$. The burden of severe hypoglycemia in type 2 diabetes. Curr Med Res Opin 2018;34:179-186.

3. Centers for Disease Control and Prevention. Estimates of diabetes and its burden in the United States. Washington, DC: US Department of Health and Human Services, 2017: $1-20$.

4. The Diabetes Control and Complication Trial Research Group. The effect of intensive treatment of diabetes on the development and progression of long-term complications in insulin-dependent diabetes mellitus. N Engl J Med 1993; 329:977-986.

5. Latts L. ADA/IBM Watson Health Study $(\mathrm{N}>300,000)$ finds that nearly $60 \%$ of people with T2D discontinue therapy after one year. Presented at: American Diabetes Association 78th Scientific Session, June 22-26, 2018, Orlando, FL.

6. National Center for Biotechnology Information; US National Library of Medicine. PubMed. https://www.ncbi.nlm.nih.gov/ pubmed/ Accessed July 18, 2018.

7. Lewis K. Humans and systems: creating natural interfaces to augment human ability. https://www.ibm.com/blogs/internetof-things/creating-natural-interfaces/ Accessed July 18, 2018.

8. Aggarwal M, Madhukar M. IBM's Watson analytics for health care: a miracle made true. Hershey, PA: IGI Global, 2016:117-134.

9. Turing Archive for the History of Computing. www .alanturing.net/turing_archive/archive/index/archiveindex .html Accessed July 18, 2018.

10. Research 2 Guidance. Top 3 therapy fields with the best market potential for digital health apps. https://research 2guidance.com/top-3-therapy-fields-with-the-best-marketpotential-for-digital-health-apps/ Accessed July 18, 2018.

11. Yeung S, Downing NL, Fei-Fei L, Milstein A. Bedside computer vision-moving artificial intelligence from driver assistance to patient safety. N Engl J Med 2018;378:12711273.

12. Young K. Newly approved software uses AI to improve diabetic retinopathy. https://www.jwatch.org/fw114063/2018/ 04/12/newly-approved-software-uses-ai-detect-diabetic Accessed July 18, 2018.

13. Pfaffl MW. A new mathematical model for relative quantification in real-time RT-PCR. Nucleic Acids Res 2001;29: $45 \mathrm{e}$.

14. López B, Martin C, Viñas PH. Special section on artificial intelligence for diabetes. Artif Intell Med 2018;85:27-28.

15. Raghupathi W, Raghupathi V. Big data analytics in healthcare: promise and potential. Health Inf Sci Syst 2014;2:3

16. Hoyt RE, Snider D, Thompson C, Mantravadi S. IBM Watson analytics: automating visualization, descriptive, and predictive statistics. JMIR Public Heal Surveill 2016;2: e157.

17. Vijan S, Hofer TP, Hayward RA. Cost-utility analysis of screening intervals for diabetic retinopathy in patients with type 2 diabetes mellitus. JAMA 2000;283:889-896.

18. Gulshan V, Peng L, Coram M, et al. Development and validation of a deep learning algorithm for detection of diabetic retinopathy in retinal fundus photographs. JAMA 2016;316:2402-2410.

19. Tariq A, Akram MU, Shaukat A, Khan SA. Automated detection and grading of diabetic maculopathy in digital retinal images. J Digit Imaging 2013;26:803-812.

20. Rahim SS, Palade V, Shuttleworth J, Jayne C. Automatic screening and classification of diabetic retinopathy fundus images. In: Mladenov V, Jayne C, Iliadis L, eds. Engineering applications of neural networks. Communications in computer and information science. Vol 459. Basel, Switzerland: Springer Nature Switzerland AG, 2014:113-122.

21. Lam C, Yu C, Huang L, Rubin D. Retinal lesion detection with deep learning using image patches. Invest Ophthalmol Vis Sci 2018;59:590-596.

22. US Food and Drug Administration. FDA permits marketing of artificial intelligence-based device to detect certain diabetes-related eye problems. https://www.fda.gov/ NewsEvents/Newsroom/PressAnnouncements/ucm604357 .htm Accessed July 18, 2018.

23. Keel S, Lee PY, Scheetz J, et al. Feasibility and patient acceptability of a novel artificial intelligence-based screening model for diabetic retinopathy at endocrinology outpatient services: a pilot study. Sci Rep 2018;8:4330.

24. Matimba A, Woodward R, Tambo E, Ramsay M, Gwanzura L, Guramatunhu S. Tele-ophthalmology: opportunities for improving diabetes eye care in resource- and specialistlimited Sub-Saharan African countries. J Telemed Telecare 2016;22:311-316.

25. Han L, Luo S, Yu J, Pan L, Chen S. Rule extraction from support vector machines using ensemble learning approach: an application for diagnosis of diabetes. IEEE J Biomed Health Inform 2015;19:728-734.

26. Shankaracharya, Odedra D, Samanta S, Vidyarthi AS. Computational intelligence-based diagnosis tool for the detection of prediabetes and type 2 diabetes in India. Rev Diabet Stud 2012;9:55-62.

27. Wei W-Q, Tao C, Jiang G, Chute CG. A high throughput semantic concept frequency based approach for patient identification: a case study using type 2 diabetes mellitus clinical notes. AMIA Annu Symp Proc 2010;2010:857861. 
28. Corey KE, Kartoun U, Zheng H, Shaw SY. Development and validation of an algorithm to identify nonalcoholic fatty liver disease in the electronic medical record. Dig Dis Sci 2016;61:913-919.

29. Neves J, Martins MR, Vilhena J, et al. A soft computing approach to kidney diseases evaluation. J Med Syst 2015; 39:131.

30. Rau H-H, Hsu C-Y, Lin Y-A, et al. Development of a webbased liver cancer prediction model for type II diabetes patients by using an artificial neural network. Comput Methods Programs Biomed 2016;125:58-65.

31. Weber C, Neeser K. Using individualized predictive disease modeling to identify patients with the potential to benefit from a disease management program for diabetes mellitus. Dis Manag 2006;9:242-256.

32. Vyas R, Bapat S, Jain E, Karthikeyan M, Tambe S, Kulkarni BD. Building and analysis of protein-protein interactions related to diabetes mellitus using support vector machine, biomedical text mining and network analysis. Comput Biol Chem 2016;65:37-44.

33. López B, Torrent-Fontbona F, Viñas R, Fernández-Real JM. Single nucleotide polymorphism relevance learning with random forests for type 2 diabetes risk prediction. Artif Intell Med 2018;85:43-49.

34. Lo-Ciganic WH, Donohue JM, Thorpe JM, et al. Using machine learning to examine medication adherence thresholds and risk of hospitalization. Med Care 2015;53:720-728.

35. Shu T, Zhang B, Yan Tang Y. An extensive analysis of various texture feature extractors to detect diabetes mellitus using facial specific regions. Comput Biol Med 2017;83: 69-83.

36. Katigari MR, Ayatollahi H, Malek M, Haghighi MK. Fuzzy expert system for diagnosing diabetic neuropathy. World $\mathbf{J}$ Diabetes 2017;8:80-88.

37. Wang L, Pedersen PC, Agu E, Strong DiM, Tulu B. Area determination of diabetic foot ulcer images using a cascaded two-wtage SVM-based classification. IEEE Trans Biomed Eng 2017;64:2098-2109.

38. Mauseth R, Lord SM, Hirsch IB, Kircher RC, Matheson DP, Greenbaum CJ. Stress testing of an artificial pancreas system with pizza and exercise leads to improvements in the system's fuzzy logic controller. J Diabetes Sci Technol 2015;9:1253-1259.

39. Ling SH, Nguyen HT. Natural occurrence of nocturnal hypoglycemia detection using hybrid particle swarm optimized fuzzy reasoning model. Artif Intell Med 2012;55: 177-184.

40. Herrero P, Pesl P, Reddy M, Oliver N, Georgiou P, Toumazou C. Advanced insulin bolus advisor based on run-torun control and case-based reasoning. IEEE J Biomed Health Inform 2015;19:1087-1096.
41. DeJournett L, DeJournett J. In silico testing of an artificialintelligence-based artificial pancreas designed for use in the intensive care unit setting. J Diabetes Sci Technol 2016;10: 1360-1371.

42. Thabit H, Tauschmann M, Allen JM, et al. Home use of an artificial beta cell in type 1 diabetes. N Engl J Med 2015; 373:2129-2140.

43. Trevitt S, Simpson S, Wood A. Artificial pancreas device systems for the closed-loop control of type 1 diabetes: what systems are in development? J Diabetes Sci Technol 2016; 10:714-723.

44. Zhang W, Yu Q, Siddiquie B, Divakaran A, Sawhney H. "Snap-n-Eat": food recognition and nutrition estimation on a smartphone. J Diabetes Sci Technol 2015;9:525-533.

45. Cvetković B, Janko V, Romero AE, Kafalı Ö, Stathis K, Luštrek M. Activity recognition for diabetic patients using a smartphone. J Med Syst 2016;40:256.

46. Wang L, Pedersen PC, Strong DM, Tulu B, Agu E, Ignotz R. Smartphone-based wound assessment system for patients with diabetes. IEEE Trans Biomed Eng 2015;62:477488.

47. Rigla M, Martínez-Sarriegui I, García-Sáez G, Pons B, Hernando ME. Gestational diabetes management using smart mobile telemedicine. J Diabetes Sci Technol 2018; 12:260-264

48. ClinicalTrails.gov. Adult accuracy study of the Elite 3 Glucose Censor (E3). https://www.clinicaltrials.gov/ct2/ show/study/NCT02246582?term $=$ NCT02246582\&rank $=1 \&$ sect $=$ X0123456 Accessed July 18, 2018.

49. Neemuchwala H. Sugar.IQ improves time-in-range by 36 mins/day, time $>180$ by 30 mins/day, time $<70$ by 6 mins/ day. Presented at: American Diabetes Association 78th Scientific Session, June 22-26, 2018, Orlando, FL.

50. Benson T. Principles of health interoperability HL7 and SNOMED, 2nd edition. London: Springer Verlag, 2012.

51. Adler-Milstein J, DesRoches CM, Kralovec P, et al. Electronic health record adoption in us hospitals: Progress continues, but challenges persist. Health Aff (Millwood) 2015;34:2174-2180.

52. Hutson TM. Missing data hinder replication of artificial intelligence studies. Www.sciencemag.org/news/2018/02/ missing-data-hinder-replication-artificial-intelligence-studies? Accessed July 18, 2018.

Address correspondence to: Marc Rivo, MD, MPH

Population Health Innovations, Inc. 4566 Prairie Avenue Miami Beach, FL 33140

E-mail: marcleerivo@gmail.com 